\title{
JPSS-1 VIIRS Pre-Launch Radiometric Performance
}

\author{
Hassan Oudrari*a, Jeff McIntire ${ }^{\mathrm{a}}$, Xiaoxiong Xiong ${ }^{\mathrm{b}}$, James Butler ${ }^{\mathrm{b}}$, Boryana Efremova ${ }^{\mathrm{a}}$, Jack \\ $\mathrm{Ji}^{\mathrm{a}}$, Shihyan Lee ${ }^{\mathrm{c}}$, Tom Schwarting ${ }^{\mathrm{a}}$

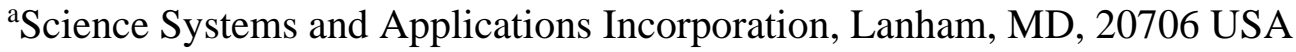 \\ bNASA Goddard Space Flight Center, Greenbelt, MD, 20771 USA. \\ 'NOAA-STAR, College Park, MD, 20740
}

\begin{abstract}
The Visible Infrared Imaging Radiometer Suite (VIIRS) on-board the first Joint Polar Satellite System (JPSS) completed its sensor level testing on December 2014. The JPSS-1 (J1) mission is scheduled to launch in December 2016, and will be very similar to the Suomi-National Polar-orbiting Partnership (SNPP) mission. VIIRS instrument was designed to provide measurements of the globe twice daily. It is a wide-swath $(3,040 \mathrm{~km})$ cross-track scanning radiometer with spatial resolutions of 370 and $740 \mathrm{~m}$ at nadir for imaging and moderate bands, respectively. It covers the wavelength spectrum from reflective to long-wave infrared through 22 spectral bands [0.412 $\mu \mathrm{m}$ to 12.01 $\mu \mathrm{m}]$. VIIRS observations are used to generate 22 environmental data products (EDRs). This paper will briefly describe J1 VIIRS characterization and calibration performance and methodologies executed during the pre-launch testing phases by the independent government team, to generate the at-launch baseline radiometric performance, and the metrics needed to populate the sensor data record (SDR) Look-Up-Tables (LUTs). This paper will also provide an assessment of the sensor pre-launch radiometric performance, such as the sensor signal to noise ratios (SNRs), dynamic range, reflective and emissive bands calibration performance, polarization sensitivity, bands spectral performance, response-vs-scan (RVS), near field and stray light responses. A set of performance metrics generated during the pre-launch testing program will be compared to the SNPP VIIRS pre-launch performance.
\end{abstract}

Keywords: VIIRS, JPSS, RSB, TEB, Calibration, Radiometric, Performance

\section{INTRODUCTION}

The purpose of this paper is to provide an overview of the initial assessment of the Visible Infrared Imaging Radiometer Suite (VIIRS) sensor onboard the Joint Polar Satellite System (JPSS). This VIIRS instrument is the second unit of its series, and it has a similar design to the first sensor that was launched on October 28, 2011 onboard the Suomi-National Polar-Orbiting Partnership (SNPP). Results summarized in this paper are based on the data analysis by NASA VIIRS Characterization Support Team (VCST), with close interaction with other government and contractor teams, including the National Aeronautics and Space Administration (NASA), the Aerospace Corporation, the National Oceanic and Atmospheric Administration (NOAA), and the University of Wisconsin.

VIIRS is a scanning radiometer that collects visible and infrared imagery and radiometric measurements of Earth's surface to study land, atmosphere, oceans and clouds, to generate environmental data in support of data continuity initiated by heritage sensors such as the Very High Resolution Radiometer (AVHRR), the Sea-viewing Wide Fieldof-view Sensor (SeaWiFS) and the Moderate Resolution Imaging Spectroradiometer (MODIS). Products derived from VIIRS will support weather forecasting, global measurements of atmospheric, oceanic, and land surface variables such as clouds, sea surface temperatures, vegetation, fire, aerosols, and ocean color. ${ }^{1}$

In the following sections, we will provide an overview of the main J1 VIIRS pre-launch radiometric and spectral performance assessment, describing briefly the main methodologies used, and comparing the results to SNPP VIIRS pre-launch performance as well as to the sensor requirements. The VIIRS spatial performance assessments are not included in this paper. J1 VIIRS design and testing program will be presented in Section 2. The summary of VIIRS 
pre-launch testing and sensor performance will be presented in section 3 for all Reflective Solar Bands (RSB) and Thermal Emissive Bands (TEB) calibration, focusing on key performance metrics such as the signal-to-noise (SNR), dynamic range, polarization, radiometric calibration uncertainties, scatter light contamination, and relative spectral response. A summary and conclusion of J1 VIIRS pre-launch radiometric and spectral performance as well as future performance enhancements will be presented in the last section, Section 4.

\section{SENSOR DESIGN AND TESTING PROGRAM}

\subsection{Sensor Design}

VIIRS instrument was designed to collect radiometric and imaging data in twenty two (22) spectral bands covering the visible and infrared spectral region between 0.4 to $12.5 \mu \mathrm{m}$ (Table 1). The moderate resolution bands (M-bands) and imaging resolution bands (I-bands) have a spatial resolution of $740 \mathrm{~m}$ and $370 \mathrm{~m}$ respectively, while the ground swath is about $3040 \mathrm{~km}$, permitting global daily coverage.

Table 1. VIIRS 22 bands characteristics and key requirements: 15 reflective bands and 7 emissive bands.

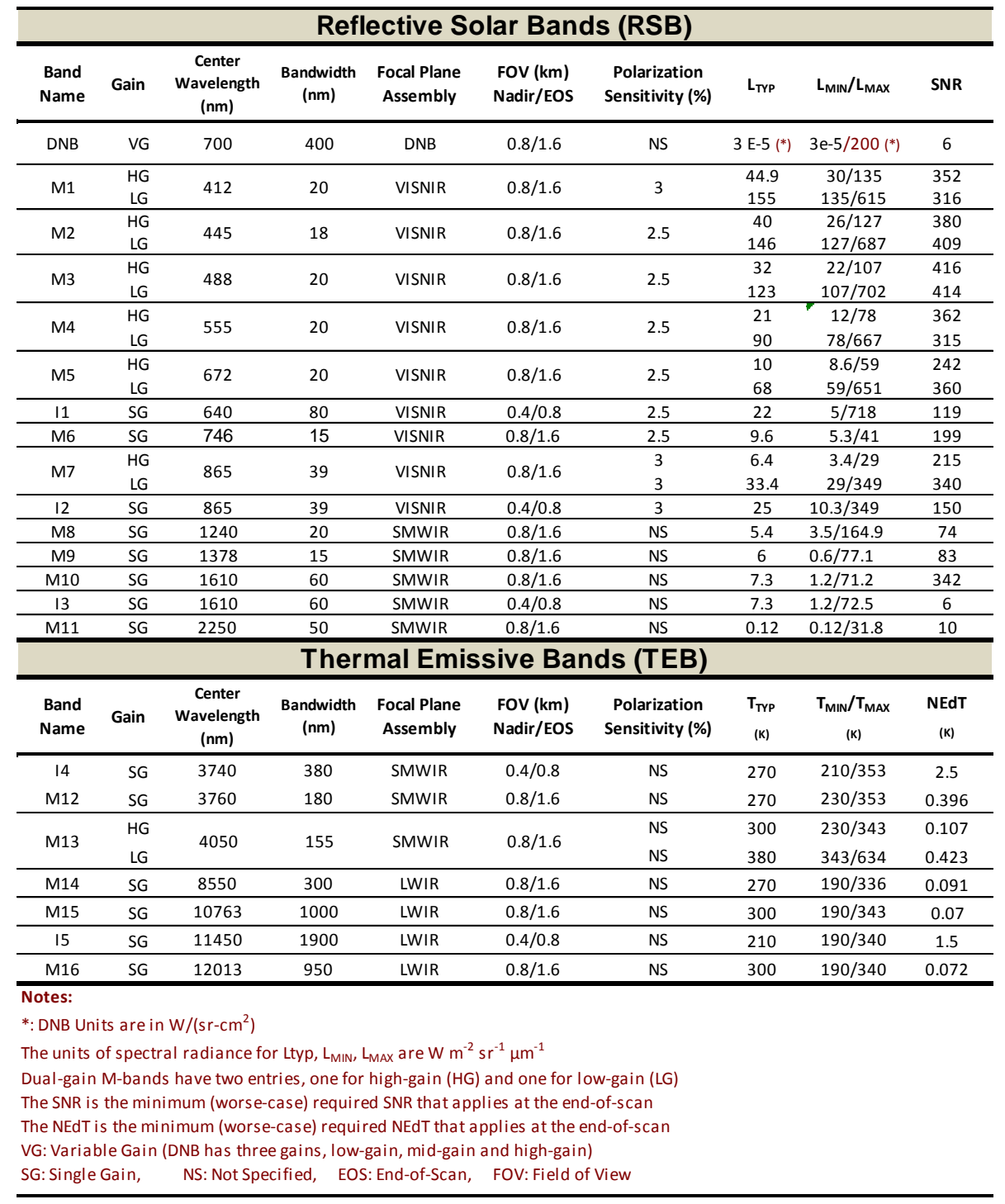


J1 VIIRS has the same design as SNPP VIIRS with similar on-board calibrators: the Spectralon ${ }^{\circledR}$ solar diffuser to calibrate the RSB, the SDSM to track the SD spectral degradation, the V-groove blackbody to calibrate the TEB, and the space view (SV) to be used for background subtraction (Figure 1).

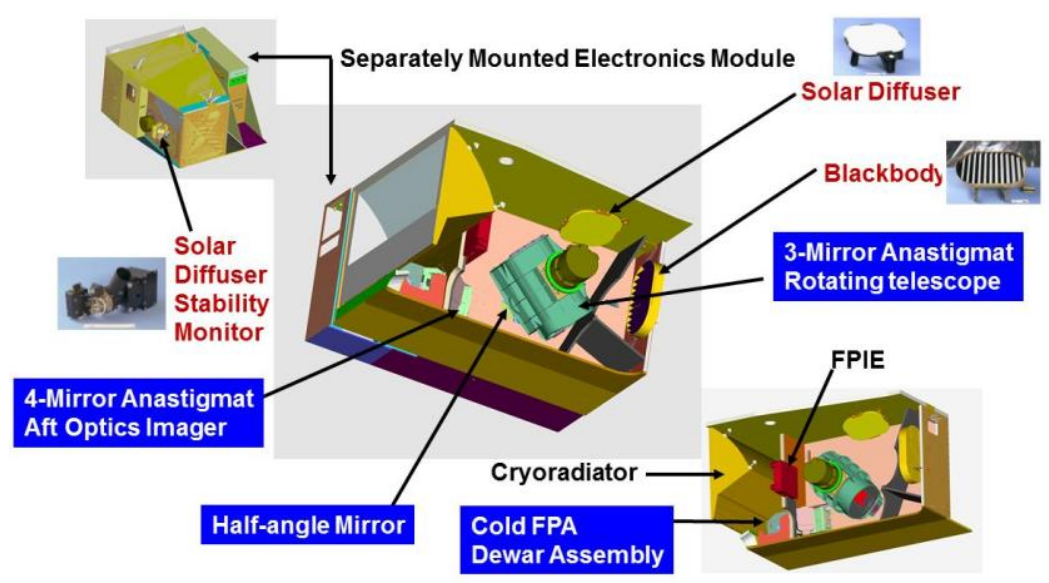

Figure 1. VIIRS instrument design and on-board calibrators: Solar Diffuser (SD), Solar Diffuser Stability Monitor (SDSM), and blackbody (BB).

VIIRS design has achieved multiple enhancements based on lessons learned from heritage sensors such as SNPP VIIRS and MODIS ${ }^{2}$. The light collected by the rotating telescope is distributed to three focal planes, the Visible Near-Infrared (VisNIR), the Short- and Mid-Wave Infrared (SMWIR), and the Long-Wave Infrared (LWIR). All bands in the SMWIR and LWIR focal planes are thermally controlled at about $80.5 \mathrm{~K}$. Light reaching the focal planes' detectors is converted into analog electric signal then digitized through analog-to-digital conversion. The VIIRS telescope scans the Earth within $\pm 56.1^{\circ}$ of nadir, then the blackbody $\left(99.57^{\circ}\right.$ to $\left.100.42^{\circ}\right)$, the $\mathrm{SD}\left(158.57^{\circ}\right.$ to $\left.159.42^{\circ}\right)$, and then the $\mathrm{SV}\left(-66.10^{\circ}\right.$ to $\left.65.25^{\circ}\right)$, in sequence.

Based on lessons learned from SNPP program, a few key changes were made to J1 instrument to enhance its performance, including: 1) the coating on the RTA mirrors was changed from Nickel (Ni) to a proprietary process (VQ) to enhance spatial stability with temperature, 2) the dichroic 2 coating was redesigned to correct the focus between SMWIR and LWIR bands, 3) the proper RTA mirror coating process was used to avoid Tungsten contamination $^{3}$ (this was an issue for SNPP once on on-orbit), and 4) the VisNIR integrated filter assembly was redesigned to significantly reduce optical scatter and out-of-bands features.

\subsection{Sensor Performance Testing}

J1 VIIRS instrument has been through a very intensive ground test program, to ensure proper characterization and calibration, to understand the instrument performance, to use reliable and approved data analysis methods, and to investigate, close and document all anomalies identified during the testing.. Multiple government funded teams were part of these characterization activities, including NASA, Aerospace Corp., NOAA, and the University of Wisconsin. The VIIRS test program, led by the sensor contractor Raytheon, provided a comprehensive sensor characterization and performance assessment over the full range of instrument operating conditions that will be encountered on orbit. It has also provided the calibration and characterization values needed for different bands, detectors, gain states, scan mirror sides, electronics configurations, and instrument temperatures. VIIRS general test plan covers three (3) key pre-launch phases: ambient (August 2013-January 2014), sensor level thermal vacuum (July-October 2014) and spacecraft thermal vacuum phase (expected in early 2016).

At the end of sensor level testing, twenty seven (27) performance test procedures were executed to characterize the instrument under various environments (ambient and/or TV), to support 141 sensor performance requirements, and to simulate long-term on-orbit performance. Key performance testing was performed at three (3) instrument plateaus 
(Cold, Nominal and Hot) which was designed to cover the range of expected on-orbit conditions. J1 VIIRS key performance testing is described in Table 2.

Early in the J1 test program, four (4) important decisions were made that will define how the instrument will be operated: 1) replacing the VIIRS 1394 communication bus with the SpaceWire to resolve the anomalies observed by SNPP on-orbit ${ }^{3}$, 2) replacing the single board computer with a new design to resolve the computer lock-up issue observed by $\mathrm{SNPP}^{3}, 3$ ) setting the cold focal planes temperature at $80.5 \mathrm{~K}$ based on the thermal balance performed at TV hot plateau, 4) and designating the VIIRS electronics side A as the primary electronics, even though both sides showed comparable performance.

Table 2: Key performance testing during Ambient and Thermal Vacuum testing phases.

\begin{tabular}{|c|c|}
\hline \multicolumn{2}{|c|}{$\begin{array}{l}\text { Ambient Phase Testing } \\
08 / 2013-01 / 2014\end{array}$} \\
\hline Polarization sensitivity & Near Filed Response (NFR) \\
\hline Response vs Scan (RVS) & Stray Light Rejection (SLR) \\
\hline Radiometric characterization: SNR, Gain & Electrical and Dynamic Crosstalk \\
\hline \multicolumn{2}{|c|}{$\begin{array}{c}\text { Thermal Vacuum Testing } \\
07 / 2014-10 / 2014\end{array}$} \\
\hline Reflective \& Emissive bands calibration & Relative Spectral Response (RSR) in-band \& OOB \\
\hline Radiometric stability & Band-to-Band Registration (BBR) \\
\hline Gain transition determination & Line spread Function (LSF) \\
\hline
\end{tabular}

\section{J1 VIIRS PRE-LAUNCH PERFORMANCE SUMMARY}

An overview of $\mathrm{J} 1$ performance assessment will be described in this section based on the NASA VCST team test data analysis. Comparison to sensor specification and in some cases to SNPP VIIRS will be presented as well. In general, data analysis methodologies are quite similar to those used for SNPP VIIRS ${ }^{2}$, except the RSB calibration, where VCST has adopted a variation to derive J1 calibration coefficients, leading to greater control of the overall calibration uncertainty determination.

\subsection{Reflective solar bands (RSB) calibration}

J1 VIIRS RSB were carefully calibrated in a TV environment using a National Institute of Standards and Technology (NIST) traceable light source, the $100 \mathrm{~cm}$ diameter Spherical Integrating Source (SIS100). Another source, the Three Mirror Collimator (TMC) SIS, capable of reaching high radiance values was also used to calibrate M1-M3 low gain. While the SIS100 has a radiance monitor to track the source fluctuations and drifts, the TMC does not, and therefore it is considered less stable than the SIS100. In addition, the space view source (SVS) was used to collect the dark offset needed to generate the background corrected detector response or digital number ( $\mathrm{dn}$ ).

The detector response is related to the light source radiance $\mathrm{L}\left(\mathrm{Wm}^{-2} \mathrm{sr}^{-1} \mathrm{um}^{-1}\right)$ through a polynomial equation:

$$
L=c_{0}+c_{1} \cdot d n+c_{2} \cdot d n^{2}+\mathrm{O}\left(d n^{3}\right)
$$

where $c_{0}, c_{1}$, and $c_{2}$ are the calibration coefficients for the quadratic part; $\mathrm{O}(\mathrm{dn} 3)$ denotes the truncation error.

The calibration coefficients can be determined from Eq. (1) through curve fitting; however, the instability of the source over time and dynamic range [Lmin, Lmax] can lead to high uncertainties of the derived radiance. To mitigate the impact of the source instability, the radiance measurements were performed with and without an attenuator inserted into the optical path. The attenuator screen is an opaque plate with small holes to allow a determined fraction of light through it. The time between attenuator in and out is very short to minimize the effect of source stability on the measurements ( 2 minutes).

The calibration equation with the attenuator screen is:

$$
\tau \cdot \mathrm{L}_{\text {attenuator }}=\mathrm{c} 0+\mathrm{c} 1 \cdot \mathrm{dn}_{\text {attenuated }}+\mathrm{c} 2 \cdot \mathrm{dn}_{\text {attenuated }^{2}}+\mathrm{O}\left(\mathrm{dn}_{\text {attenuated }}{ }^{3}\right),
$$


where $\tau$ is the transmittance of attenuator and $\mathrm{L}_{\text {attenuator }}$ is the reference radiance when the attenuator is deployed.

Eqs. $(1,2)$ can be combined and approximated by:

$$
\frac{\tau \cdot L_{\text {attenuator }}}{L} \approx \tau=\frac{h_{0}+d n_{\text {attenuated }}+h_{2} \cdot d n_{\text {attenuated }}^{2}}{h_{0}+d n+h_{2} \cdot d n^{2}},
$$

where $\mathrm{L}_{\text {attenuator }}$ is the radiance with the attenuator screen, $\mathrm{dn}_{\text {attenuated }}$ is the attenuated response, $\tau$ is the attenuator transmission, $h_{0}=c_{0} / c_{1}$, and $h_{2}=c_{2} / c_{1}$.

The two ratios of calibration coefficients $h_{0}$ and $h_{2}$ can be derived regardless of how well the absolute quantity of the reference radiance is known as long as the light source remains stable or its variability is corrected. The parameters

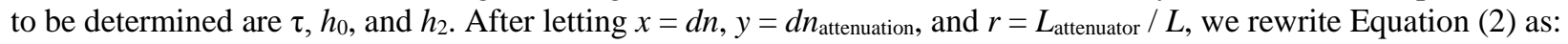

$$
\frac{\tau \cdot r \cdot x-y}{1-\tau \cdot r}=h_{0}+h_{2} \frac{y^{2}-\tau \cdot r \cdot x^{2}}{1-\tau \cdot r} .
$$

The parameter $r$ represents a correction factor for the SIS radiance drift between measurements. The parameter $h_{0}$ in Equation (3) can be eliminated by subtracting a pair of measurements while $h_{2}$ can be eliminated by a division of two sets of the subtracted results. The statistical value of $\tau$ is derived using combinations of 3 or 4 measurements from the data set of all radiance levels.

After $\tau$ has been determined independently, a linear fit of Equation (3) gives $h_{0}$ as the intercept and $h_{2}$ as the slope. Finally, rearranging the truncated calibration equation we have:

$$
c_{1}=\frac{L_{\mathrm{SIS} 100}}{h_{0}+d n+h_{2} \cdot d n^{2}} .
$$

The error analysis of calibration is straightforward. For $f=h_{0}+d n+h_{2} \cdot d n^{2}$, the relative uncertainty is:

$$
\frac{\Delta f}{f}=\frac{\left(1+2 h_{2} d n\right) \cdot \Delta d n}{h_{0}+d n+h_{2} \cdot d n^{2}} .
$$

Our findings have confirmed the very small non-linearity term for all RSB $\left(\sim 10^{-6}\right)$, and the coefficients are strongly dependent on the data selection in the fitting process. The RSB calibration coefficients derivation based on this new method will be described and discussed in more details in a separate paper $^{4}$, and have shown comparable values to the baseline method. However, this method has the advantage of providing more insight into the quality of the fitting and more flexibility in the uncertainty calculation.

\section{SNR Calculation}

The detector SNR was calculated for each SIS-100 radiance and for each sample position based on a moving window (24 samples) to eliminate artifacts due to source non-uniformity, by dividing the sample averaged dn over scans by the standard deviation as:

$$
S N R=\frac{1}{M} \sum_{j=1}^{j=M}\left[\frac{\frac{1}{N}\left[\sum_{i=1}^{i=N} d n_{i . j}\right]}{\sigma_{j}}\right]
$$

where $\mathrm{M}, \mathrm{N}$ are the total number of samples (in the window) and scans respectively, and $\mathrm{i}, \mathrm{j}$ are the number of scans and samples respectively. A mathematical equation was used to facilitate the computation of the SNR at any radiance level and to smooth out the variability in the SNR over radiance levels.

The band averaged SNR and radiance saturation values are shown in table 3. All J1 RSB meet the SNR specification at $\mathrm{L}_{\text {typ }}$ with good margins, but these margins are a little smaller than those measured for SNPP. It is also worth noting that without the mirror coating contamination in the SNPP RTA, J1 SNR performance is expected to be better 
than SNPP at the end of mission life. The smallest and largest SNR margins for J1 are 31\% and 2567\% for M5 and $\mathrm{I} 3$, respectively. It is also worth mentioning that the I3 detector 4 is a very noisy detector, and its responsivity is about $50 \%$ lower than the other detectors in this band.

RSB saturation is comparable to SNPP, meeting specification with acceptable margins, except for bands M8 (72\%) and I3 (92\%). These two Lmax non-compliances were expected and have small effect on science based on SNPP experience.

Table 3: J1 VIIRS SNR and radiance saturation values for reflective bands

\begin{tabular}{|c|c|c|c|c|c|c|c|c|c|}
\hline Band & $\begin{array}{c}\text { Gain } \\
\text { Stage }\end{array}$ & $\begin{array}{c}\text { SNR } \\
\text { (Spec) }\end{array}$ & $\begin{array}{c}\text { Lmax } \\
\text { (Spec) }\end{array}$ & $\begin{array}{c}\text { SNPP } \\
\text { SNR }\end{array}$ & $\begin{array}{c}\text { J1 } \\
\text { SNR }\end{array}$ & $\begin{array}{c}\text { SNPP } \\
\text { SNR/Spec }\end{array}$ & $\begin{array}{c}\text { J1 } \\
\text { SNR/Spec }\end{array}$ & $\begin{array}{c}\text { SNPP _sat/Lmax } \\
\text { L_sat/Lmax }\end{array}$ \\
\hline M1 & H & 352 & 135 & 613 & 566 & 1.74 & 1.61 & 1.16 & 1.21 \\
\hline M1 & L & 316 & 615 & 1042 & 956 & 3.30 & 3.03 & 1.13 & 1.10 \\
\hline M2 & H & 380 & 127 & 554 & 514 & 1.46 & 1.35 & 1.41 & 1.40 \\
\hline M2 & L & 409 & 687 & 963 & 861 & 2.35 & 2.11 & 1.20 & 1.30 \\
\hline M3 & H & 416 & 107 & 683 & 630 & 1.64 & 1.51 & 1.29 & 1.31 \\
\hline M3 & L & 414 & 702 & 1008 & 868 & 2.44 & 2.10 & 1.20 & 1.20 \\
\hline M4 & H & 362 & 78 & 526 & 486 & 1.45 & 1.34 & 1.42 & 1.39 \\
\hline M4 & L & 315 & 667 & 864 & 695 & 2.74 & 2.21 & 1.31 & 1.28 \\
\hline M5 & H & 242 & 59 & 373 & 316 & 1.54 & 1.31 & 1.24 & 1.25 \\
\hline M5 & L & 360 & 651 & 776 & 591 & 2.16 & 1.64 & 1.12 & 1.11 \\
\hline M6 & H & 199 & 41 & 409 & 382 & 2.06 & 1.92 & 1.16 & 1.16 \\
\hline M7 & H & 215 & 29 & 524 & 467 & 2.44 & 2.17 & 1.28 & 1.26 \\
\hline M7 & L & 340 & 349 & 721 & 595 & 2.12 & 1.75 & 1.19 & 1.17 \\
\hline M8 & H & 74 & 164.9 & 358 & 273 & 4.84 & 3.69 & 0.77 & 0.72 \\
\hline M9 & H & 83 & 77.1 & 290 & 271 & 3.49 & 3.27 & 1.09 & 1.04 \\
\hline M10 & H & 342 & 71.2 & 691 & 627 & 2.02 & 1.83 & 1.14 & 1.09 \\
\hline M11 & H & 10 & 31.8 & 105 & 23 & 10.49 & 2.30 & 1.09 & 1.10 \\
\hline I1 & H & 119 & 718 & 261 & 184 & 2.19 & 1.55 & 1.07 & 1.08 \\
\hline I2 & H & 150 & 349 & 273 & 251 & 1.82 & 1.67 & 1.18 & 1.17 \\
\hline I3 & H & 6 & 72.5 & 176 & 154 & 29.36 & 25.67 & 0.97 & 0.91 \\
\hline
\end{tabular}

\section{SWIR Non-Linearity Issue}

J1 SWIR M-bands exhibited more non-linear behavior at low radiance than what was seen on SNPP. Figure 2 shows the ratios of attenuator out signal to attenuator in signal for SWIR bands M8-M11. The non-constant dn ratio reflects the large non-linear response at low radiance levels (SNPP dn ratios were much flatter). An investigation of this issue discovered that an ASP bias voltage for the SMWIR M-bands, VR_Clamp, was set to a different voltage value $(-0.2 \mathrm{~V})$ compared to its setting on SNPP $(-0.4 \mathrm{~V})$. The non-linearity present in the SWIR M-bands produces large errors in the attenuator ratios, and therefore large errors in the radiance characterization uncertainty and uniformity. A mitigation plan is being prepared to enhance the calibration performance of the SWIR bands, based on either a third degree polynomial equation or the adoption of a two-piece calibration (i.e. calibration over two radiance ranges).

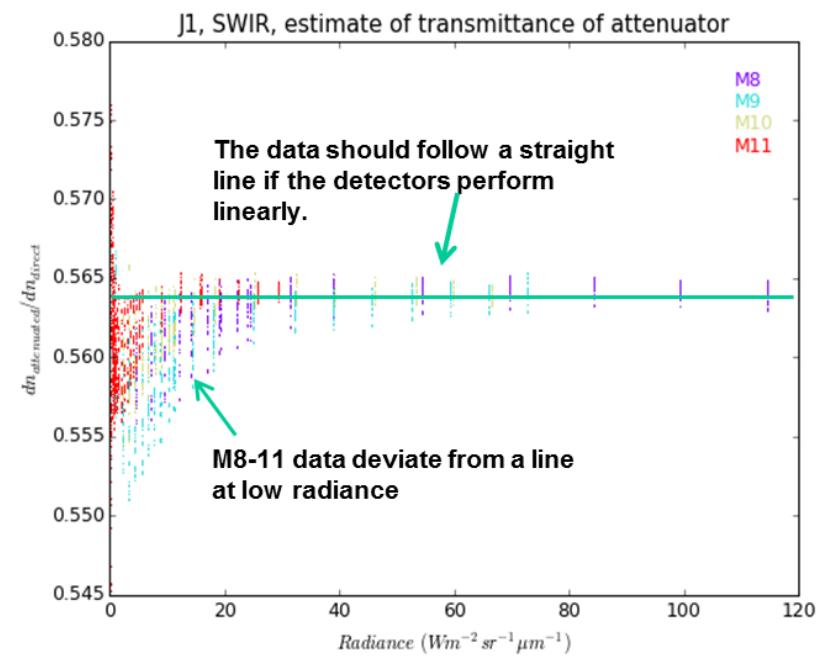


Figure 2: J1 SWIR non-linearity: ratio of sensor response value (dn) for attenuator in and out

\section{RSB calibration uncertainties}

VIIRS RSB requirements on the absolute radiometric calibration are against the spectral reflectance accuracy of $2 \%$ when viewing a uniform scene of typical radiance, $\mathrm{L}_{\text {TYP. }}$. This uncertainty verification was performed using contributors which have been constrained by sensor-level requirements while others are based on the sensor contractor allocations. A contributor to the radiometric uncertainty includes the detector response characterization uncertainty, which states that the response of a detector to a range of radiance levels from $\mathrm{L}_{\mathrm{MIN}}$ to $\mathrm{L}_{\mathrm{MAX}}$ must be fit with a second degree polynomial to within $0.3 \%$. This characterization uncertainty requirement was also very challenging for the VIIRS RSB to meet, but the spectral reflectance accuracy ( $2 \%)$ was met for all RSB except M11 $(2.25 \%)^{5}$, primarily due to the uncertainty of the SD bi-directional reflectance factor (BRF).

\subsection{Thermal emissive bands (TEB) calibration}

J1 VIIRS thermal band calibration is referenced to a NIST traceable external Blackbody Calibration Source (BCS). The radiance reaching the detector is the sum of the source radiance as well as contributors along the optical path (i.e. the RTA, HAM, and aft optics). The reflectance factors represent the total reflectance of the RTA mirrors and aft-optics. The RVS is the scan angle dependent, relative reflectance of the HAM. The radiances of the sources are determined via Planck's law convolved over the relative spectral response (RSR) of each spectral band over the extended band pass. The temperature of each source is determined from one or more thermistors.

The path difference radiance between the two sources (BCS and SV) is then

$\Delta L_{B C S}=R V S_{B C S} \varepsilon_{B C S} L_{B C S}+\frac{\left(R V S_{B C S}-R V S_{S V}\right)}{\rho_{R T A}}\left[L_{H A M}+\left(1-\rho_{R T A}\right) L_{R T A}\right]$.

The path difference at-detector radiance is modeled as a quadratic polynomial in the offset corrected digital response, or $\Delta L=c_{0}+c_{1} d n+c_{2} d n^{2}$.

The retrieved EV radiance for the BCS is determined by inverting Eq. (8), or

$$
L_{B C S-r e t}=\frac{\left(c_{0}+c_{1} d n_{B C S}+c_{2} d n_{B C S}^{2}\right)}{R V S_{B C S}}-\frac{\left(R V S_{B C S}-R V S_{S V}\right)}{R V S_{B C S} \rho_{R T A}}\left[L_{H A M}+\left(1-\rho_{R T A}\right) L_{R T A}\right] \text {. }
$$

The low gain state of band M13 cannot be calibrated using the BCS like the high gain state, so a second, high temperature external blackbody (TMC BB) was used. The calibration of the TMC BB was tied to the BCS by cross calibration at lower scene temperatures where both sources overlap.

\section{NEdT calculation}

The NEdT is the fluctuation in the scene temperature equivalent to the system noise and is computed via the equation

$$
N E d T=\frac{\Delta L_{B C S}}{S N R \frac{\partial L}{\partial T}}
$$

The derivative is of Planck's law with respect to the source temperature. The NEdT was determined at all source levels and compared to the specified value at $\mathrm{T}_{\mathrm{TYP}}$; this was determined using the fit $\mathrm{SNR}$ and at-detector radiance. The band average NEdT results are below specification as shown in Table 4. All emissive bands and detectors are meeting the NEdT specification with good margins, and performance is comparable to SNPP. A mild dependency of NEdT to sensor temperature was confirmed. The smallest and greatest margins were observed for M14 (39\%) and I4 $(595 \%)$ respectively. Detector variability is small in general, with the exception of detector 4 in M15 and detector 5 in M16B which are out-of-family detectors. 
Table 4 also shows the J1 maximum temperature and comparison to specification and SNPP. All bands have saturation values above the specified Lmax, and comparable to SNPP. Two bands have analog saturation before digital saturation, M12 and I4, and have roll-over post-saturation. The TEB saturation values are consistent among electronics sides and temperature plateaus to within $3 \mathrm{~K}$. M13 low gain saturation was not observed in TV testing, but saturation is expected around $670 \mathrm{~K}$ based on ambient testing.

Table 4: J1 VIIRS Lmax and NEdT performance for TEB

\begin{tabular}{|l|l|l|l|l|l|l|}
\hline \multirow{2}{*}{ Band } & \multicolumn{3}{|c|}{ Lmax } & \multicolumn{3}{c|}{ NEdT at Ttyp } \\
\cline { 2 - 7 } & Spec & SNPP & \multicolumn{1}{c|}{ J1 } & Spec & SNPP & \multicolumn{1}{c|}{ J1 } \\
\hline 14 & 353 & 357 & 357 & 2.5 & 0.41 & 0.42 \\
\hline 15 & 340 & 373 & 370 & 1.5 & 0.42 & 0.41 \\
\hline M12 & 353 & 357 & 358 & 0.396 & 0.13 & 0.12 \\
\hline M13 HG & 343 & 364 & 363 & 0.107 & 0.044 & 0.043 \\
\hline M13 LG & 634 & - & - & 0.423 & 0.34 & 0.304 \\
\hline M14 & 336 & 347 & 348 & 0.091 & 0.061 & 0.05 \\
\hline M15 & 343 & 365 & 359 & 0.07 & 0.03 & 0.026 \\
\hline M16 & 340 & 368 & 369 & 0.072 & 0.038 & 0.043 \\
\hline
\end{tabular}

The radiometric response uniformity (RRU) represents the detector-to-detector uniformity (or striping) and is quantified by the following equation:

$$
R R U=\frac{\left|L_{B C S-r e t}-\left\langle L_{B C S-r e t}\right\rangle_{D}\right|}{N E d L},
$$

where the average EV retrieved radiance is over all detectors in a band and NEdL is derived from Eq. (11). The sensor specification is met if the RRU is less than unity within the radiance range from $\mathrm{L}_{\mathrm{MIN}}$ to $0.9 \mathrm{~L}_{\mathrm{MAX}}$.

Figure 3 shows the detector-to-detector striping performance represented by the RRU metric. RRU values larger than one mean that the sensor is not compliant with detector striping requirements. We can easily see that the risk of striping increases with temperature for all TEB bands, because the deviation of the detector retrieved radiance from the band average increases with temperature while the NEdL levels off. Results derived per HAM side, electronics side and temperature plateau have shown some performance variations, with RRU for some band reaching up to 2 at highest temperatures (larger than $310 \mathrm{~K}$ ). We should also emphasize the difficulty to meet this requirement for both SNPP and J1 since VIIRS TEB has a very good noise performance (very low NEdL).

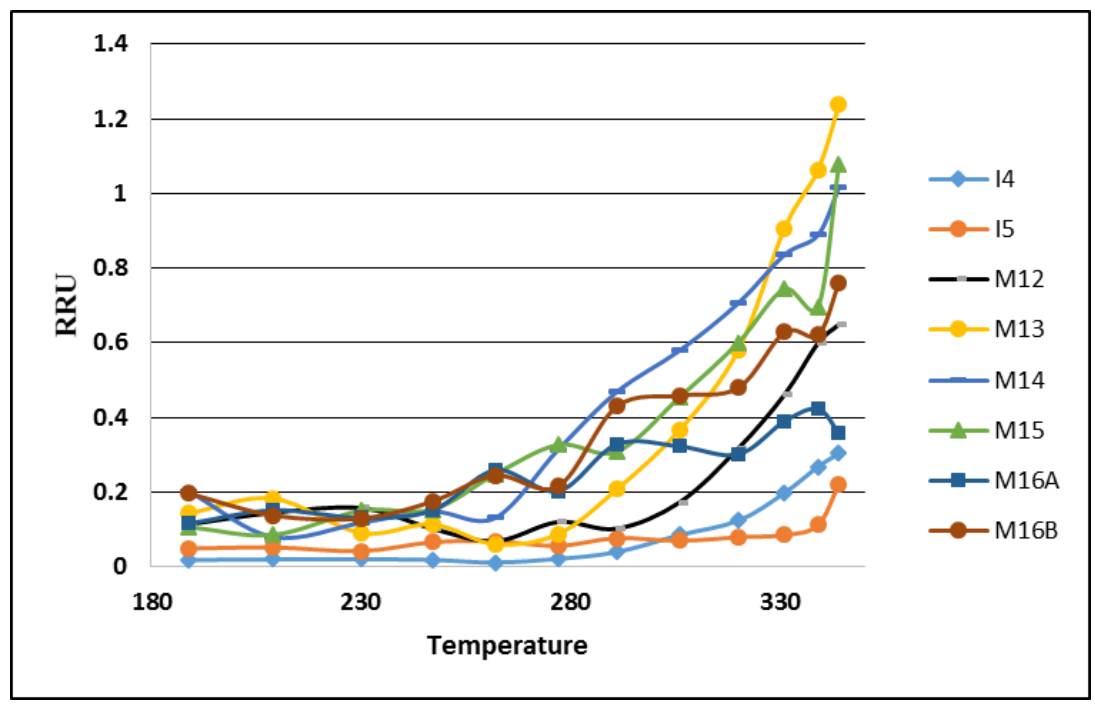


Figure 3. J1 maximum RRU as a function of source (BCS) temperature is shown for all TEB, for electronics side A, HAM A, and Nominal plateau.

The Absolute Radiance Difference (ARD) is the percent difference between the retrieved and BCS radiances, and it is essentially a measure of the fit uncertainty's effect on the accuracy of the retrieved radiance, or

$$
A R D=100 \frac{L_{B C S-r e t}-L_{B C S}}{L_{B C S}} .
$$

The band averaged ARD for J1 TEB derived at nominal plateau, electronics side A are shown in table 5 alongside the specified values in parenthesis. As expected, the ARD results show excellent thermal calibration for all bands at the specified temperatures, and the only exception is M12 at very low temperature $(230 \mathrm{~K})$ where the ARD value of 7.6 is slightly higher the specified value of 7.0. Again, the ARD performance is comparable between J1 and SNPP.

Table 5. Band averaged ARD at specified temperature shown for all TEB, for electronics side A, HAM A, and Nominal plateau. ARD specification values are in parenthesis.

\begin{tabular}{|c|c|c|c|c|c|c|c|c|}
\hline \multicolumn{9}{|c|}{ ARD Performance (\%) } \\
\hline Temp (K) & 14 & 15 & M12 & M13 & M14 & M15 & M16A & M16B \\
\hline 190 & $\sim$ & $\sim$ & $\sim$ & $\sim$ & $0.68(12.30)$ & $0.29(2.10)$ & $0.17(1.60)$ & $0.25(1.60)$ \\
\hline 230 & $\sim$ & $\sim$ & $7.60(7.00)$ & $2.95(5.7)$ & $0.11(2.40)$ & $0.07(\mathbf{0 . 6 0 )}$ & $0.08(\mathbf{0 . 6 0 )}$ & $0.04(0.60)$ \\
\hline 267 & $0.48(5.00)$ & $0.10(2.50)$ & $\sim$ & $\sim$ & $\sim$ & $\sim$ & $\sim$ & $\sim$ \\
\hline 270 & $\sim$ & 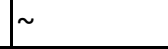 & $0.24(0.70)$ & $0.15(\mathbf{0 . 7 0})$ & $0.08(0.60)$ & $0.05(\mathbf{0 . 4 0})$ & $0.04(0.40)$ & $0.04(\mathbf{0 . 4 0})$ \\
\hline 310 & $\sim$ & $\sim$ & $0.25(\mathbf{0 . 7 0})$ & $0.17(0.70)$ & $0.11(0.40)$ & $0.06(0.40)$ & $0.03(\mathbf{0 . 4 0 )}$ & $0.04(0.40)$ \\
\hline 340 & $\sim$ & $\sim$ & $0.27(0.70)$ & $0.18(0.70)$ & $0.09(\mathbf{0 . 5 0 )}$ & $0.05(\mathbf{0 . 4 0 )}$ & $0.03(\mathbf{0 . 4 0 )}$ & $0.03(\mathbf{0 . 4 0})$ \\
\hline
\end{tabular}

\subsection{Other Performance Testing}

\section{Polarization}

During ambient phase testing, J1 polarization sensitivity was characterized using an integrating sphere combined with a sheet polarizer at seven different scan angles in the 400 to $900 \mathrm{~nm}$ range covering all VisNIR bands ${ }^{6,7}$. The sheet polarizer was mounted on a rotary stage and was rotated in 15 degree increments from 0 to 360 degrees. Additionally, a long wavelength spectral blocking filter was placed in the optical path when measuring the short wavelength bands, M1 to M3, to eliminate near infrared Out-Of-Band (OOB) contributions. The polarization sensitivity was derived for all VisNIR bands, detectors, HAM sides, and seven scan angles (-55, -45, -22, -8, 20, 45, 55), using Fourier series. The quality of $\mathrm{J} 1$ measurements based on the Fourier transform is good, since only the zeroth and second order terms have shown non-negligible results. The polarization sensitivity factors derived based on ambient testing have revealed unexpectedly large non-compliance for four bands, M1-M4. This polarization issue was linked to the redesigned VisNIR spectral filters, and confirmed by the sensor polarization model ${ }^{6,7}$. Based on these findings further performance testing was performed in the post-TV phase adding more scan-angles $(-37,-30,-$ $15,4)$ and a limited testing with monochromatic source for two bands, M1 and M4, to verify the quality of the sensor polarization modeling ${ }^{6}$.

The final J1 mean polarization factors are shown for all VisNIR bands in Table 6 for HAM side A. The polarization maximum and specification values per band are also shown. The linear polarization sensitivity for bands M1 - M4 was observed to be higher than the specified limit, as high as $\sim 5.4 \%$ for M1, $4.2 \%$ for M2, $2.9 \%$ for M3, and $\sim 4.2 \%$ for M4. Differences in linear polarization sensitivity with HAM side are as high as $\sim 1 \%$, where HAM side $\mathrm{B}$ is generally larger. Large detector to detector and scan angle differences were observed with bands (up to $\sim 4 \%$ in M1). This is likely the result of angle of incidence changes on the VisNIR spectral filter assembly. Special testing conducted after nominal testing (post-TV phase) confirmed the high quality of J1 polarization characterization test 
data, identified the spectral filter as the root cause of the anomaly, and indicated that the sensor polarization model predictions are acceptable but still shows some difference with measurements, especially for M4.

Table 6: Overall VisNIR linear polarization factor for both J1 and SNPP sensors

\begin{tabular}{|c|c|c|c|c|c|c|c|c|c|c|c|c|c|c|}
\hline \multirow{2}{*}{ Band } & \multirow{2}{*}{ Sensor } & \multicolumn{11}{|c|}{ Scan Angle } & \multirow[b]{2}{*}{ Max Pol. } & \multirow[b]{2}{*}{ Spec } \\
\hline & & -55 & -45 & -37 & -30 & -22 & -15 & -8 & 4 & 20 & 45 & 55 & & \\
\hline \multirow{2}{*}{ I1 } & SNPP & 1.5 & 1.24 & $\sim$ & $\sim$ & 0.93 & $\sim$ & 0.85 & $\sim$ & 0.7 & 0.64 & 0.62 & 1.24 & 2.5 \\
\hline & J1 & 0.81 & 0.74 & 0.75 & 0.73 & 0.73 & 0.79 & 0.76 & 0.8 & 0.82 & 0.85 & 0.85 & 0.85 & 2.5 \\
\hline \multirow{2}{*}{12} & SNPP & 0.29 & 0.27 & $\sim$ & $\sim$ & 0.34 & $\sim$ & 0.37 & $\sim$ & 0.47 & 0.51 & 0.51 & 0.51 & 3 \\
\hline & J1 & 0.73 & 0.62 & 0.54 & 0.47 & 0.36 & 0.37 & 0.37 & 0.43 & 0.5 & 0.61 & 0.66 & 0.62 & 3 \\
\hline \multirow{2}{*}{ M1 } & SNPP & 2.99 & 2.63 & $\sim$ & $\sim$ & 1.95 & $\sim$ & 1.79 & $\sim$ & 1.42 & 1.21 & 1.4 & 2.63 & 3 \\
\hline & J1 & 5.13 & 5.26 & 5.35 & 5.52 & 5.54 & 5.56 & 5.65 & 5.7 & 5.66 & 5.51 & 5.37 & 5.7 & 3 \\
\hline \multirow{2}{*}{ M2 } & SNPP & 2.11 & 1.97 & $\sim$ & $\sim$ & 1.63 & $\sim$ & 1.53 & $\sim$ & 1.28 & 1.17 & 1.29 & 1.97 & 2.5 \\
\hline & J1 & 3.72 & 3.79 & 3.85 & 3.95 & 3.9 & 3.89 & 3.94 & 3.95 & 3.9 & 3.99 & 4.04 & 3.99 & 2.5 \\
\hline \multirow{2}{*}{ M3 } & SNPP & 1.2 & 1.14 & $\sim$ & $\sim$ & 0.9 & $\sim$ & 0.82 & $\sim$ & 0.61 & 0.7 & 0.8 & 1.14 & 2.5 \\
\hline & J1 & 2.89 & 2.85 & 2.83 & 2.85 & 2.73 & 2.69 & 2.68 & 2.63 & 2.62 & 2.8 & 2.84 & 2.85 & 2.5 \\
\hline \multirow{2}{*}{ M4 } & SNPP & 1.05 & 1.1 & $\sim$ & $\sim$ & 1.19 & $\sim$ & 1.16 & $\sim$ & 1 & 0.88 & 0.84 & 1.19 & 2.5 \\
\hline & J1 & 3.61 & 3.9 & 4.08 & 4.16 & 4.17 & 4.22 & 4.18 & 4.18 & 4.04 & 3.89 & 3.8 & 4.22 & 2.5 \\
\hline \multirow{2}{*}{ M5 } & SNPP & 1.19 & 1.02 & $\sim$ & $\sim$ & 0.85 & $\sim$ & 0.84 & $\sim$ & 0.76 & 0.73 & 0.69 & 1.02 & 2.5 \\
\hline & J1 & 1.9 & 1.86 & 1.9 & 1.86 & 1.82 & 1.85 & 1.79 & 1.83 & 1.81 & 1.8 & 1.8 & 1.9 & 2.5 \\
\hline \multirow{2}{*}{ M6 } & SNPP & 0.99 & 0.96 & $\sim$ & $\sim$ & 0.94 & $\sim$ & 0.94 & $\sim$ & 0.88 & 0.82 & 0.76 & 0.96 & 2.5 \\
\hline & J1 & 1.62 & 1.32 & 1.13 & 0.99 & 0.86 & 0.85 & 0.79 & 0.75 & 0.73 & 0.75 & 0.76 & 1.32 & 2.5 \\
\hline \multirow{2}{*}{ M7 } & SNPP & 0.17 & 0.19 & $\sim$ & $\sim$ & 0.25 & $\sim$ & 0.28 & $\sim$ & 0.38 & 0.42 & 0.41 & 0.42 & 3 \\
\hline & J1 & 0.73 & 0.62 & 0.54 & 0.46 & 0.36 & 0.36 & 0.32 & 0.39 & 0.45 & 0.55 & 0.6 & 0.62 & 3 \\
\hline
\end{tabular}

\section{Relative Spectral Response}

VIIRS sensor level spectral testing was performed using a monochromator source in ambient for VisNIR bands, and in the TV environment at nominal plateau for TEB bands. Additional RSR testing was performed for VisNIR bands in the post-TV phase using a laser source. $\mathrm{J} 1$ spectral characterization was completed successfully, and analysis completed by the government team based on the SNR and visual inspection identified the highest quality measurements. Figure 4 shows the in-band response for M1, M8 and M16. As noted previously, the redesign of the VisNIR filters led to major enhancements in terms of OOB reduction as shown in the figure 5 where we compare the full M1 RSR between J1 and SNPP sensors. Compliance with the band center, bandwidth, extended band pass, and Integrated $\mathrm{OOB}$ (IOOB) requirements were calculated for all bands on a per detector basis. Table 7 shows the compliance matrix for J1 and SNPP spectral requirements, and we can easily observe the enhancements of J1 IOOB for the VisNIR bands compared to SNPP. Most other non-compliances are either similar to SNPP or not considered a risk to the SDR or EDR performance. Further enhancement to the VisNIR RSRs is expected before J1 instrument launch, by combining best quality measurements from testing performed using the monochromator source and the laser source.

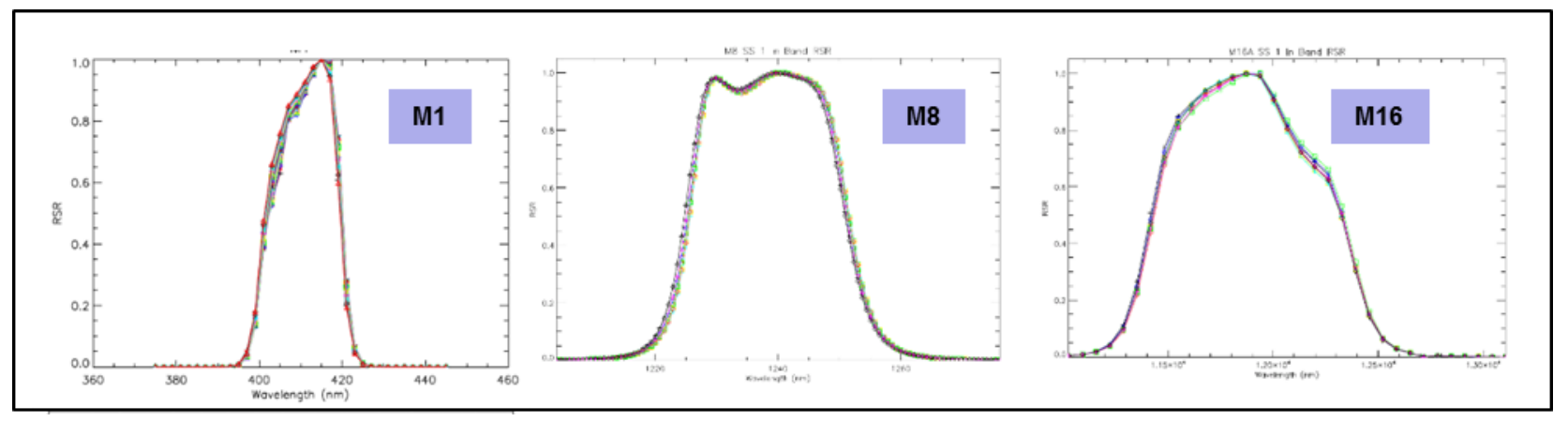


Figure 4: J1 RSR for bands M1, M8 and M16 derived during sensor level testing
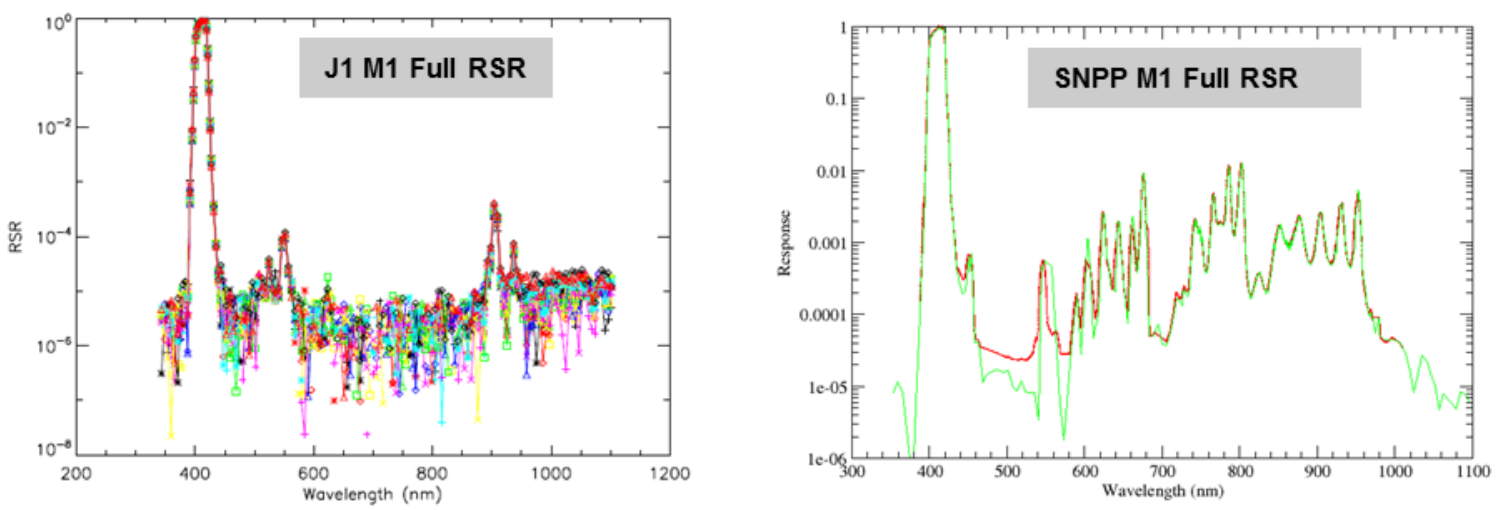

Figure 5: M1 full RSR comparison between J1 and SNPP

Table 7: J1 Spectral Performance Summary (left table), and comparison to SNPP (right table)

\begin{tabular}{|c|c|c|c|c|c|c|c|c|c|c|c|}
\hline \multirow[b]{2}{*}{ Band } & \multicolumn{3}{|c|}{ SNPP } & \multirow[b]{2}{*}{$\begin{array}{c}\text { Upper 1\% } \\
\text { point }\end{array}$} & \multirow[b]{2}{*}{ МІООВ } & \multirow[b]{2}{*}{ Band } & \multirow[b]{2}{*}{$\begin{array}{l}\text { Band } \\
\text { center }\end{array}$} & \multicolumn{2}{|c|}{ J1 } & \multirow[b]{2}{*}{$\begin{array}{l}\text { Upper } 1 \% \\
\text { point }\end{array}$} & \multirow[b]{2}{*}{ MIOOB } \\
\hline & $\begin{array}{l}\text { Band } \\
\text { center }\end{array}$ & $\begin{array}{l}\text { Bandpass } \\
\text { (FWHM) }\end{array}$ & $\begin{array}{c}\text { Lower } 1 \% \\
\text { point }\end{array}$ & & & & & $\begin{array}{l}\text { Bandpass } \\
\text { (FWHM) }\end{array}$ & $\begin{array}{l}\text { Lower 1\% } \\
\text { point }\end{array}$ & & \\
\hline 'M1' & pass & pass & pass & pass & FAIL & 'M1' & pass & FAIL & pass & pass & pass \\
\hline 'M2' & pass & FAIL & pass & pass & pass & 'M2' & pass & pass & pass & pass & pass \\
\hline 'M3' & pass & pass & pass & pass & FAIL & 'M3' & pass & pass & pass & pass & pass \\
\hline 'M4' & FAIL & pass & pass & pass & FAIL & 'M4' & pass & pass & pass & pass & pass \\
\hline 'I1' & pass & pass & pass & pass & pass & 'I1' & pass & pass & Pass & pass & pass \\
\hline 'M5' & pass & pass & pass & pass & FAIL & 'M5' & FAIL & pass & pass & pass & pass \\
\hline 'M6' & pass & pass & pass & pass & FAIL & 'M6' & pass & pass & pass & pass & pass \\
\hline 'I2' & pass & pass & pass & pass & FAIL & 'I2' & pass & pass & pass & pass & pass \\
\hline 'M7' & pass & pass & pass & pass & pass & 'M7' & pass & pass & pass & pass & pass \\
\hline 'M8' & pass & FAIL & pass & pass & pass & 'M8' & pass & FAIL & pass & pass & pass \\
\hline 'M9' & pass & pass & pass & pass & pass & 'M9' & pass & pass & pass & pass & pass \\
\hline 'I3' & pass & pass & pass & pass & pass & 'I3' & pass & pass & pass & pass & pass \\
\hline 'M10' & pass & pass & pass & pass & pass & 'M10' & pass & pass & pass & pass & pass \\
\hline 'M11' & pass & pass & pass & pass & pass & 'M11' & pass & pass & pass & pass & pass \\
\hline '14' & pass & pass & pass & pass & pass & '14' & pass & pass & pass & pass & pass \\
\hline 'M12' & pass & pass & pass & pass & pass & 'M12' & pass & pass & pass & pass & pass \\
\hline 'M13' & pass & pass & pass & pass & pass & 'M13' & pass & pass & pass & pass & pass \\
\hline 'M14' & pass & FAIL & pass & pass & FAIL* & 'M14' & pass & FAIL & pass & pass & pass \\
\hline 'M15' & pass & pass & pass & pass & FAIL* & 'M15' & pass & pass & pass & pass & FAIL* \\
\hline 'I5' & pass & pass & pass & FAIL & FAIL* & '15' & pass & pass & pass & FAIL & FAIL* \\
\hline 'M16A' & FAIL & pass & pass & pass & FAIL* & 'M16A' & FAIL & FAIL & pass & pass & FAIL* \\
\hline 'M16B' & FAIL & pass & pass & pass & FAIL* & 'M16B' & FAIL & pass & pass & pass & FAIL* \\
\hline DNBLGS & pass & pass & pass & pass & pass & DNBLGS & pass & FAIL & FAIL & pass & pass \\
\hline
\end{tabular}

\section{Response Versus Scan-angle}

The RVS testing was performed during ambient phase for the RSB using the SIS-100 and for the TEB using a cavity type blackbody and the on-board BB. Data taken at eleven angle-of-incidence (AOI) for RSB and twelve for TEB were used to fit the RVS function, which is a quadratic polynomial in AOI, after corrections for source drift and background radiances were performed.

Figure 6 shows J1 band-average RVS functions for HAM side A. For all bands, the RVS differences between HAM sides are small with the exception of band M6. The variation in the RSB RVS for M1-M11 and I1-I3 is generally small and similar to SNPP, varying by less than $1.5 \%$ (M1 has largest variation) over the full operational AOI range of $28.6^{\circ}$ to $60.2^{\circ}$. The RSB RVS uncertainty requirement is $0.3 \%$ which was determined here as the fitting residuals. All RSB bands had uncertainties lower than $0.1 \%$ except band M9 which was about $0.2 \%$. The determination of band M9 RVS was complicated due to water vapor fluctuations during RVS testing. The TEB RVS is generally small varying by less than $1 \%$ over the full AOI range. In contrast, the LWIR RVS changes by up to $10 \%$ for M14 
over the range of AOI. The TEB RVS uncertainty requirement is $0.2 \%$, which was determined here as the fitting residuals; all TEB RVS uncertainties are lower than $0.15 \%$, meeting the RVS characterization uncertainty requirement with good margin. Overall, J1 RVS performance is comparable to SNPP performance.
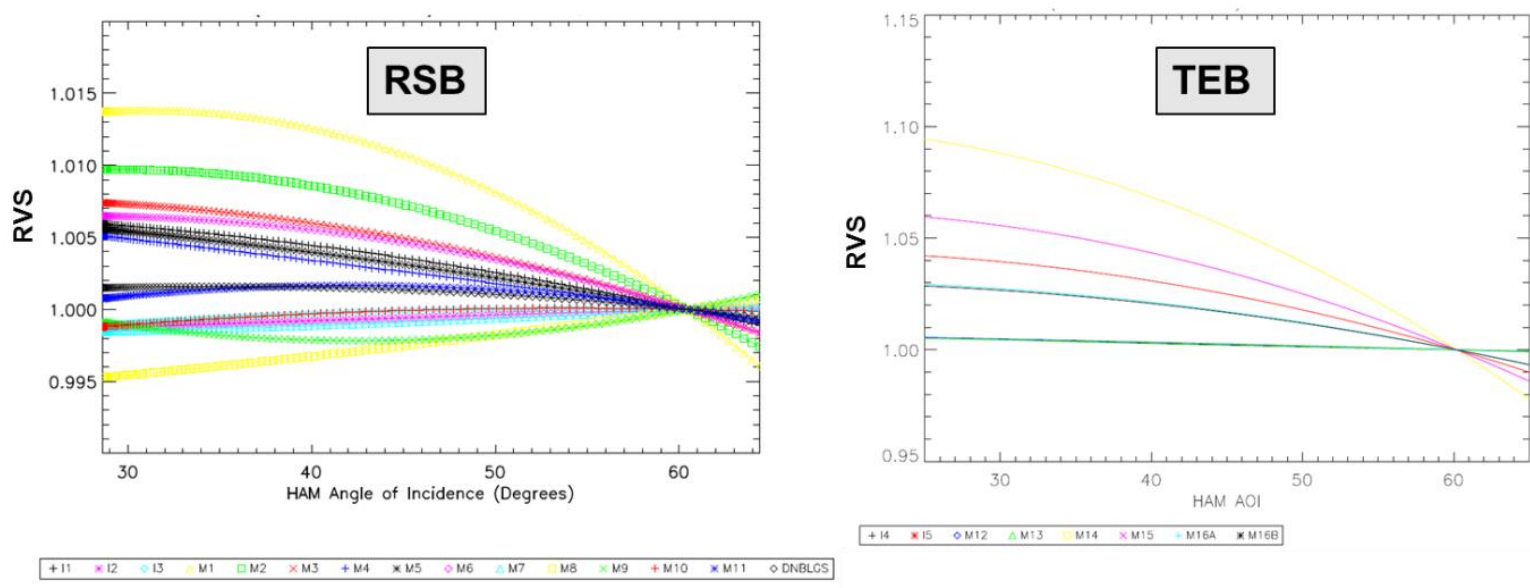

Figure 6: J1 VIIRS band averaged response vs. scan-angle (RVS) as a function AOI for RSB and TEB

\section{Near Field Response}

Near field response (NFR) is defined as scattered light within 4 degrees from the RTA line of sight. The Scatter Measurement Assembly (ScMA) light source was used with a slit and a band pass filter to measure the sensor NFR. The VIIRS NFR requirement is defined as structured scene performance specified as the maximum allowable response at a specified angle limit coming off a 20 by $20 \mathrm{~km}$ bright target for each band. To estimate the structured scene response, a Harvey-Shack BRDF scattering model was used to fit the measured NFR profile and remove test artifacts and noisy samples.

Figure 7 shows the normalized response for band M5 detector 8 (SNPP and J1) which represents a typical profile observed in the VIIRS NFR measurements when the sensor is scanning the source through a vertical slit reticle. For both SNPP and J1, the figure shows the NFR falling off rapidly from the peak with additional sharp drops seen around the field baffle locations. In this example, the M5 NFR drops to about $0.1 \%$ within 5 samples of the peak (i.e. corresponding to approximately $1.25 \mathrm{~km}$ at the Earth surface), and the field baffle reduces the NFR by about one order. The NFR is estimated for each detector, and the results show good detector uniformity within the same band. The NFR requirement states that the maximum allowable scattered radiance, as a fraction of typical scene radiance, from a bright target at the specified angular distance shall be less than the specified value. The J1 band averaged NFR performance is compliant for all bands, and is as good as SNPP in general.

SNPP

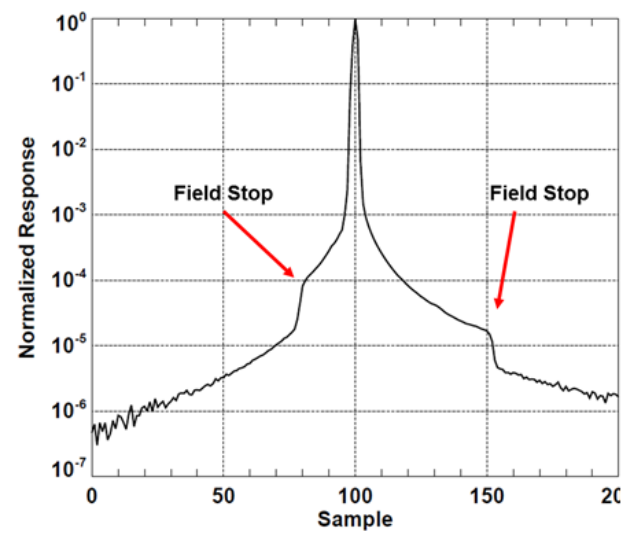

J1

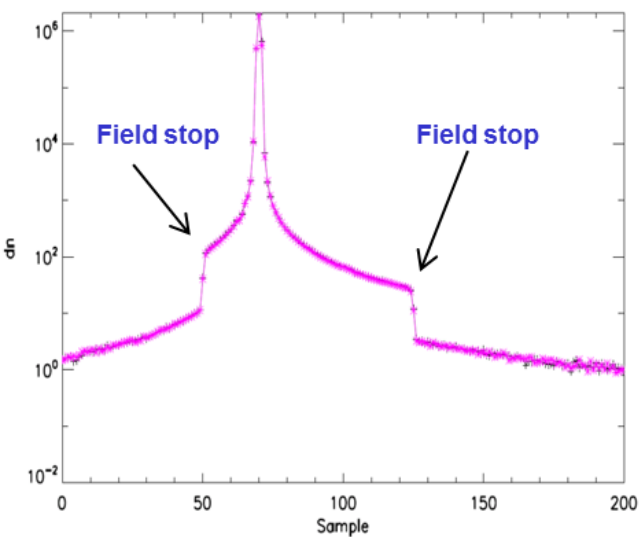


Figure 7: J1 Measured NFR for band M5 $(672 \mathrm{~nm})$ detector 8, as a function of samples. The figure also shows the comparison to SNPP NFR performance.

\section{Stray Light Response}

The far field stray light is defined as a region between 4 and 62.5 degrees from the sensor boresight. The far field stray light testing was performed using a radiometrically calibrated 1000-W studio lamp. The VIIRS telescope was staring at a cavity type blackbody while the lamp was moved through 33 equal-distanced hemispherical positions to evaluate the angular stray light distribution. The lamp positions covered roughly equally divided annulus out to 62.5 degrees off nadir to simulate the Earth as subtended by VIIRS at the J1 on-orbit operating altitude. The measured instrument response was scaled by the ratio of the studio lamp irradiance and a model of bright cloud irradiance. The VIIRS far-field stray light requirement uses a worse case, hole-in-the-cloud scenario. The measured instrument response at each position was weighted by the corresponding annulus then aggregated to estimate the total stray light contribution from the specified worse-case scenario. The test was conducted with the RTA locked at SV, nadir, and end of scan to obtain stray light estimates at different scan angles.

Figure 8 shows the estimated J1 VIIRS far field stray light compliance. The sensor test data analysis showed all RSB meet the stray light requirement with large margins at the beginning of life. Margins are between $50 \%$ (M5) and $95 \%$ (I3). Furthermore, test results indicate that the stray light rejection is comparable between J1 and SNPP. A noticeable difference is that the stray light in SNPP is much stronger along track than along scan direction; whereas in J1, the stray light is more evenly distributed in all angles. Another difference is that band M11 is now compliant for J1 because of Ltyp requirement change (from 0.1 to 1.0).

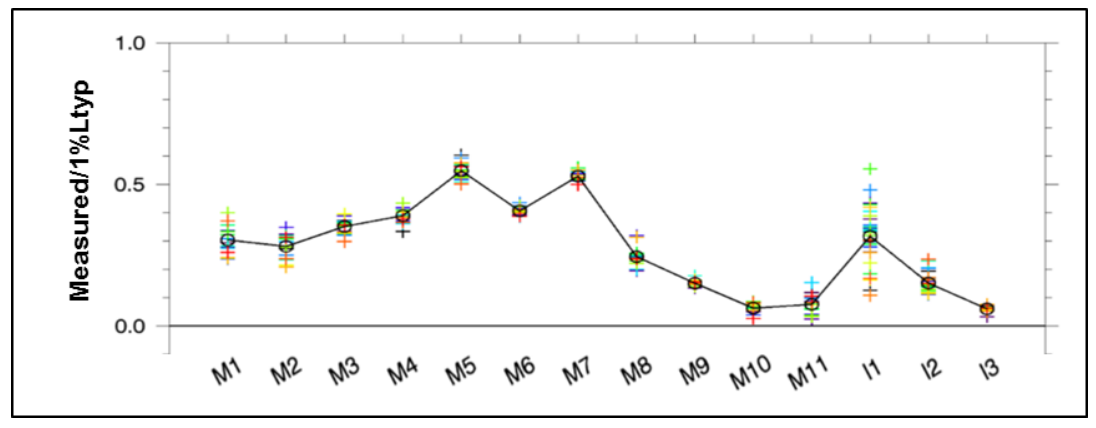

Figure 8: Summary of J1 VIIRS stray light performance. The ratio of measured stray light to requirement (1\% Ltyp) is less than 1 (i.e. requirement compliance). Symbols represent 16 detectors.

\section{SUMMARY}

This paper provided an early assessment of the J1 VIIRS pre-launch radiometric performance, based on an intensive testing program that was designed by the sensor contractor with support from the government calibration and science team. The instrument testing program was performed to characterize the radiometric, spectral and spatial performance for all 22 spectral bands in various configurations and environments, and simulating the range of onorbit conditions (ambient and thermal vacuum). The key radiometric and spectral performances were discussed in this paper. The VIIRS spectral bands calibration and characterization have shown very good performance in term of SNR, NEdT, dynamic range, gain transition, relative spectral response, and scattered light. Overall, J1 performance was comparable to SNPP VIIRS. Minor issues related to polarization and non-linearity of SWIR bands were well characterized and could be adjusted for in the ground processing system, leading to high quality products.

\section{Acknowledgments}

The authors of this paper would like to thank the VIIRS team members from NOAA, Aerospace Corporation, NASA Science Team, University of Wisconsin and Raytheon for their valuable contributions to VIIRS testing and 
performance verifications. We also want to thank MODIS characterization and Support Team (MCST) as well as previous VCST members, who provided valuable support to the VIIRS program and to the pre-launch calibration and characterization effort.

\section{References}

[1] Lottman, B. T., Mussetto, M. S., McCarthy, J. K., Emch, P. G., Shipley, S. T., St. Germain, K., Mango, S. A., and Guenther, B. W., "NPP/NPOESS Joint Government SSPR Contractor Calibration and Validation program: system perspective, cooperative strategy and path to NPP", Proc. SPIE 5549, 20 (2004)

[2] Oudrari, H., McIntire, J., Xiong, X., Butler, J., Lee, S., Lei, N., Schwarting, T., and Sun, J., "Prelaunch Radiometric Characterization and Calibration of the S-NPP VIIRS Sensor," Geoscience and Remote Sensing, IEEE Transactions on , vol.53, no.4, pp.2195,2210, April 2015, doi: 10.1109/TGRS.2014.2357678

[3] Xiong, X., Oudrari, H., Chiang, K., McIntire, J., Fulbright, J., Lei, N., Sun, J., Efremova, B., Wang, Z., and Butler, J., "VIIRS on-orbit calibration activities and performance", IGARSS, Australia, July 2013

[4] Ji, Q., Efremova, B., Schwarting, T., McIntire, J., Oudrari, H., and Xiong, X., "A robust method for determining quadratic calibration coefficients for VIIRS reflective solar bands", In press, Proc. of SPIE (2015).

[5] Joint Polar Satellite System (JPSS) VIIRS Reflective Solar Bands - Performance Verification Report (PVR), Goddard Space Flight Center, Greenbelt, MD, USA, 2011.

[6] McIntire, J., Young, J. B., Moyer, D., Waluschka, E., Oudrari, H., and Xiong, X., "Analysis of JPSS J1 VIIRS Polarization Sensitivity Using the NIST T-SIRCUS”, In press, Proc. of SPIE (2015).

[7] Waluschka, E., McCorkel, J., McIntire, J., Moyer, D., McAndrew, B., Brown, S.W., Lykke, K., Young, J.B., Fest, E., Butler, J., Wang, T.R., Monroy, E.O., Turpie, K., Meister. G., Thome, K.J., J1 VIIRS polarization narrative: testing and performance, In press, Proc. of SPIE (2015). 